\title{
Stitching-free 3D printing of millimeter-sized highly transparent spherical and aspherical optical components
}

\author{
Simon RistoK, ${ }^{1,}{ }^{*}$ (1) Simon Thiele, ${ }^{2}$ Andrea Toulouse, ${ }^{2}$ Alois \\ M. Herkommer, ${ }^{2}$ ANd HARALd Giessen ${ }^{1}$ \\ ${ }_{1}^{14 t h}$ Physics Institute and Research Center SCoPE, University of Stuttgart, Pfaffenwaldring 57, 70569 \\ Stuttgart, Germany \\ ${ }^{2}$ Institute of Applied Optics (ITO) and Research Center SCoPE, University of Stuttgart, Pfaffenwaldring 9, \\ 70569 Stuttgart, Germany \\ *s.ristok@pi4.uni-stuttgart.de
}

\begin{abstract}
We demonstrate the fabrication of optical elements on the millimeter scale by stitching-free 3D printing via two-photon polymerization, using a commercial microfabrication system (Nanoscribe $\mathrm{GmbH}$ ). Previous limitations are overcome by the use of a large writing field objective as well as a novel high transparency resist. The printed optical components are free of stitching defects due to a single step exposure and exhibit an unpreceded glass-like appearance due to the low absorption of the resist material throughout the entire visible wavelength range. We print aspherical focusing lenses, characterize and optimize their shape fidelity, and find their optical performance close to the simulated optimum. For comparison with commercially available glass lenses we also fabricate spherical half-ball lenses of different sizes. The imaging quality of the lenses is very similar, underpinning the powerfulness of our fabrication strategy.
\end{abstract}

(C) 2020 Optical Society of America under the terms of the OSA Open Access Publishing Agreement

\section{Introduction}

Optical elements with millimeter size are nowadays standard components in many technical devices, ranging from smartphone cameras over industrial and medical endoscopes to numerous optical sensors used in the automotive industry. Optical designs can be straightforwardly scaled down from the centimeter to the millimeter scale, which is, due to scaling laws, in favor for aberration control; their fabrication, however, can pose significant challenges. While spherical glass lenses are readily available down to $0.3 \mathrm{~mm}$ diameter, complex lens systems required for more elaborate applications are difficult to scale, as they cannot be manufactured by grinding or milling. In these cases, polymer lenses fabricated by different 3D printing techniques have emerged as an interesting alternative [1-5]. Direct laser writing based on two-photon polymerization is not only able to create arbitrary free-form surfaces but also offers excellent intrinsic alignment of multi-component lenses [6-10]. The diameter of 3D printed optical elements fabricated by this technique ranges from tens of micrometers to several hundreds of micrometers [11-18], leaving a size gap around the millimeter scale to the aforementioned standard fabrication techniques. The challenge thus is pushing the size of the printed structures from the micrometer into the millimeter realm. This is in principal possible by dividing a larger lens into smaller units which are printed sequentially, however, the stitching marks between adjacent units deteriorate the imaging quality. Advanced fabrication techniques can reduce the influence of stitching marks [19], however, it is more desirable to print a lens in a single step without stitching. Furthermore, the increased size and volume of the lenses reveals one of the downsides of the commonly used materials: they often retain a yellow color after polymerization due to residual photoinitiator, which also has negative influence on the imaging quality, in particular in structures with large volumes. 
In this work, we overcome these challenges with the help of two distinct advances: The use of a commercially available objective with a large writing field in combination with a new commercially available photoresist with low absorption and low luminescence in the visible wavelength range [20]. This combination enables us to 3D print millimeter-sized lenses without stitching marks and unpreceded optical clarity. We demonstrate spherical and aspherical lenses with $1 \mathrm{~mm}$ diameter as well as spherical lenses with $2 \mathrm{~mm}$ diameter. All lenses are printed directly on glass coverslips and consist of a single curved surface. The lenses are characterized in terms of shape fidelity, imaging quality, and modulation transfer function.

\section{Fabrication}

The 3D printer used for the fabrication is a Nanoscribe Photonic Professional GT. A liquid photoresist is hardened by laser-induced two-photon polymerization. The laser is focused by a microscope objective, generating a small polymerized region called a voxel (volume pixel). By moving the voxel through the resist, arbitrary 3D structures can be created. We use the system in dip-in laser lithography (DILL) mode, where the resist is drop-coated on a glass coverslip and the focusing objective is directly immersed into the resist. The coverslip is exposed to an oxygen plasma to increase the adhesion of the polymerized structures. Prior to the printing process, the 3D model of the printed structure is decomposed into slices, each slice consisting of many parallel hatching lines. For each slice the voxel is scanned along the lines using a pair of galvanometric mirrors. After a slice is finished, the distance between objective and substrate is increased and the next slice is printed. The increase in distance between slices is called slicing distance, the distance
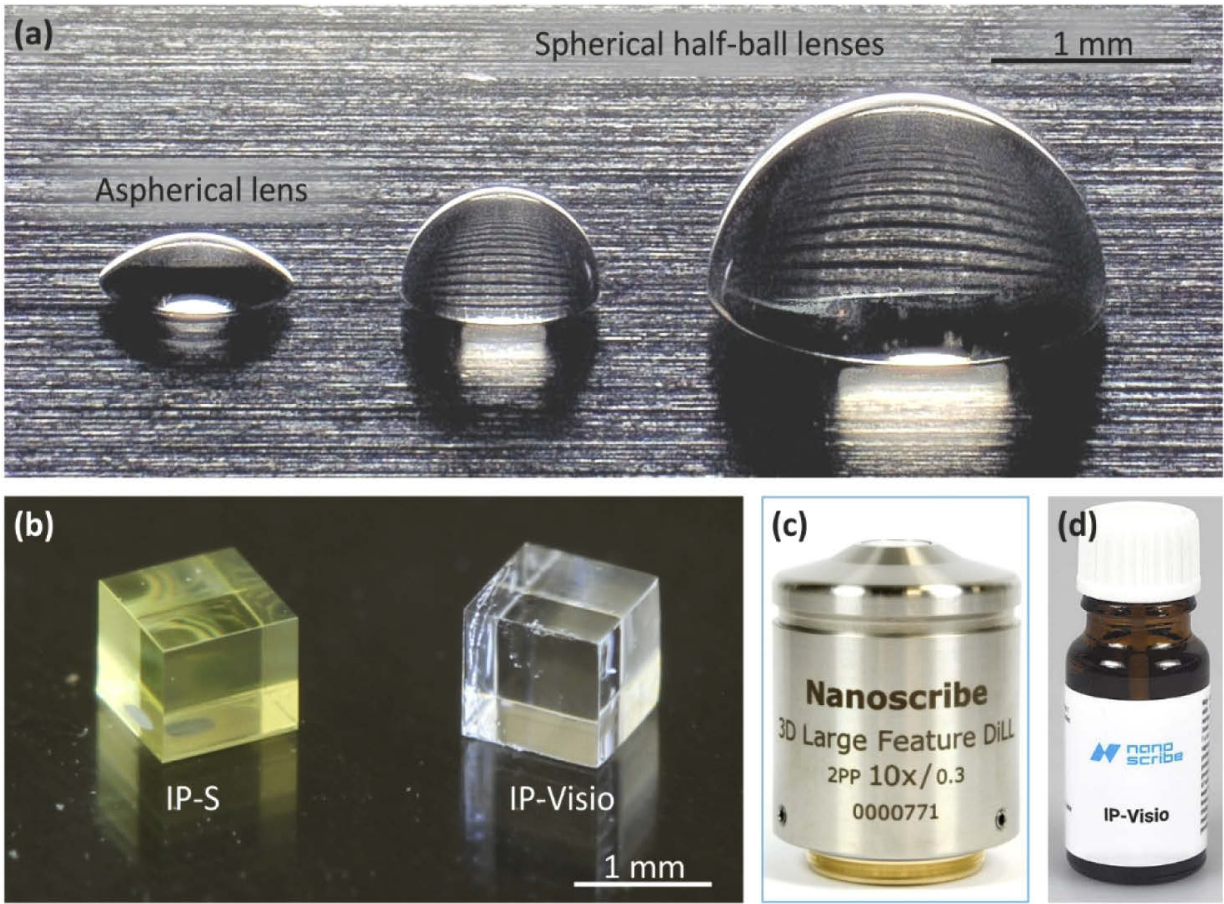

Fig. 1. Overview of printed lenses and components used for 3D printing. (a) Aspheric lens with $1 \mathrm{~mm}$ diameter and half-ball lenses with $1 \mathrm{~mm}$ and $2 \mathrm{~mm}$ diameter. (b) 3D printed cubes with $1 \mathrm{~mm}$ side length, made from IP-S and IP-Visio. (c) 10x objective with large writing field diameter. Image courtesy of Nanoscribe GmbH. (d) Photoresist IP-Visio used for all printed lenses. Image courtesy of Nanoscribe $\mathrm{GmbH}$. 
between the parallel lines in a slice is called hatching distance. After the printing process is finished, the remaining liquid photoresist is dissolved by immersing the coverslip in a developing solution (mr-Dev 600, micro resist technology) for $25 \mathrm{~min}$ and subsequently in isopropyl alcohol for $5 \mathrm{~min}$. After drying with nitrogen, the substrate is placed on a hot plate at $60{ }^{\circ} \mathrm{C}$ for $1 \mathrm{~h}$ and is simultaneously illuminated by UV light to increase the degree of polymerization.

An overview of the printed lenses under investigation is displayed in Fig. 1(a). The aspheric lens on the left is designed to demonstrate the superior focusing ability of non-spherical lenses with free-form surfaces. The lens diameter is $1 \mathrm{~mm}$. The half-ball lenses in the middle and on the right have diameters of $1 \mathrm{~mm}$ and $2 \mathrm{~mm}$ and are printed to compare them to commercially available glass lenses with the same shape and size. The resist used for the lenses is called IP-Visio (Nanoscribe GmbH, Fig. 1(d)) and was recently introduced. Compared to other resists for two-photon polymerization, e.g., IP-S (Nanoscribe GmbH), the yellow color is significantly reduced. This is particularly obvious in Fig. 1(b), where cubes with $1 \mathrm{~mm}$ side length made from IP-S (left) and IP-Visio (right) are displayed side-by-side. The picture was taken 9 months after fabrication, which demonstrates that the high optical quality does not deteriorate over time. During this time, the sample was stored in ambient conditions. The 10x objective used for the fabrication is depicted in Fig. 1(c). It has a numerical aperture (NA) of 0.3, a working distance of $700 \mu \mathrm{m}$ and a specified writing field diameter of $1 \mathrm{~mm}$. The lenses presented in this work are printed with a slicing distance of $1.5 \mu \mathrm{m}$ and a hatching distance of $0.5 \mu \mathrm{m}$. The laser power parameter is set to $100 \%$ and the laser focus is moved in lateral direction at a scan speed of 50 $\mathrm{mm} / \mathrm{s}$. The writing time is $3 \mathrm{~h}$ for the aspheric lens, $5 \mathrm{~h}$ for the $1 \mathrm{~mm}$ half-ball lens, and $23 \mathrm{~h}$ for the $2 \mathrm{~mm}$ half-ball lens.

\section{Aspherical focusing lens}

Spherical glass lenses with diameters ranging from $0.3 \mathrm{~mm}$ up to several millimeters are standard products nowadays, e.g., ball-lenses for fiber coupling. While they are well-suited for certain applications, better results can often be achieved by introducing non-spherical surfaces into the optical system. In order to illustrate this, a spherical and an aspherical lens with identical center thicknesses and diameters are compared in Fig. 2(a) and (b). The superior focusing capability of the aspheric design is obvious due to correction of spherical aberration. The importance of aspherical components increases even more when we look at more complex optical systems with multiple optical elements. Being able to create non-spherical lens shapes is therefore one of the crucial advantages of $3 \mathrm{D}$ printing.

We fabricate an aspherical lens corresponding to the design from Fig. 2(b). A $170 \mu \mathrm{m}$ thick coverslip is used as substrate and is included in the optical design. First, a baseplate with a diameter of $1.2 \mathrm{~mm}$ and a thickness of $50 \mu \mathrm{m}$ is printed, which is required for subsequent shape characterization. Then, the actual lens with a diameter of $1 \mathrm{~mm}$ and a center thickness of $227 \mu \mathrm{m}$ is printed on top of the baseplate. In order to achieve the designed spot size it is essential that the deviation of the printed lens from the optical design is minimized. Therefore, we characterize the lens surface using a confocal microscopy technique (Nanofocus $\mu$ surf expert). We fabricate four identical lenses and extract two profiles per lens, using the flat area of the baseplate as reference plane. In Fig. 2(c) the mean profile (black) is compared to the optical design (red). The printed lenses are on average $15 \mu \mathrm{m}$ thinner in the center than designed, which results from shrinking effects during the polymerization and the subsequent developing step. The radial dependence of the shape deviation is depicted in black in Fig. 2(d). We use a polynomial fitting function to determine the shape of the deviation. The fitting function is added to the original optical design and the lens is reprinted. This procedure is repeated iteratively to optimize the shape fidelity. The surface profile after two iterations (blue) in Fig. 2(c) matches the optical design much better than the first printed lens (black). Over the main part of the lens $(r<450 \mu \mathrm{m})$ the residual deviation (Fig. 2(d), blue) is smaller than $1 \mu \mathrm{m}$. 

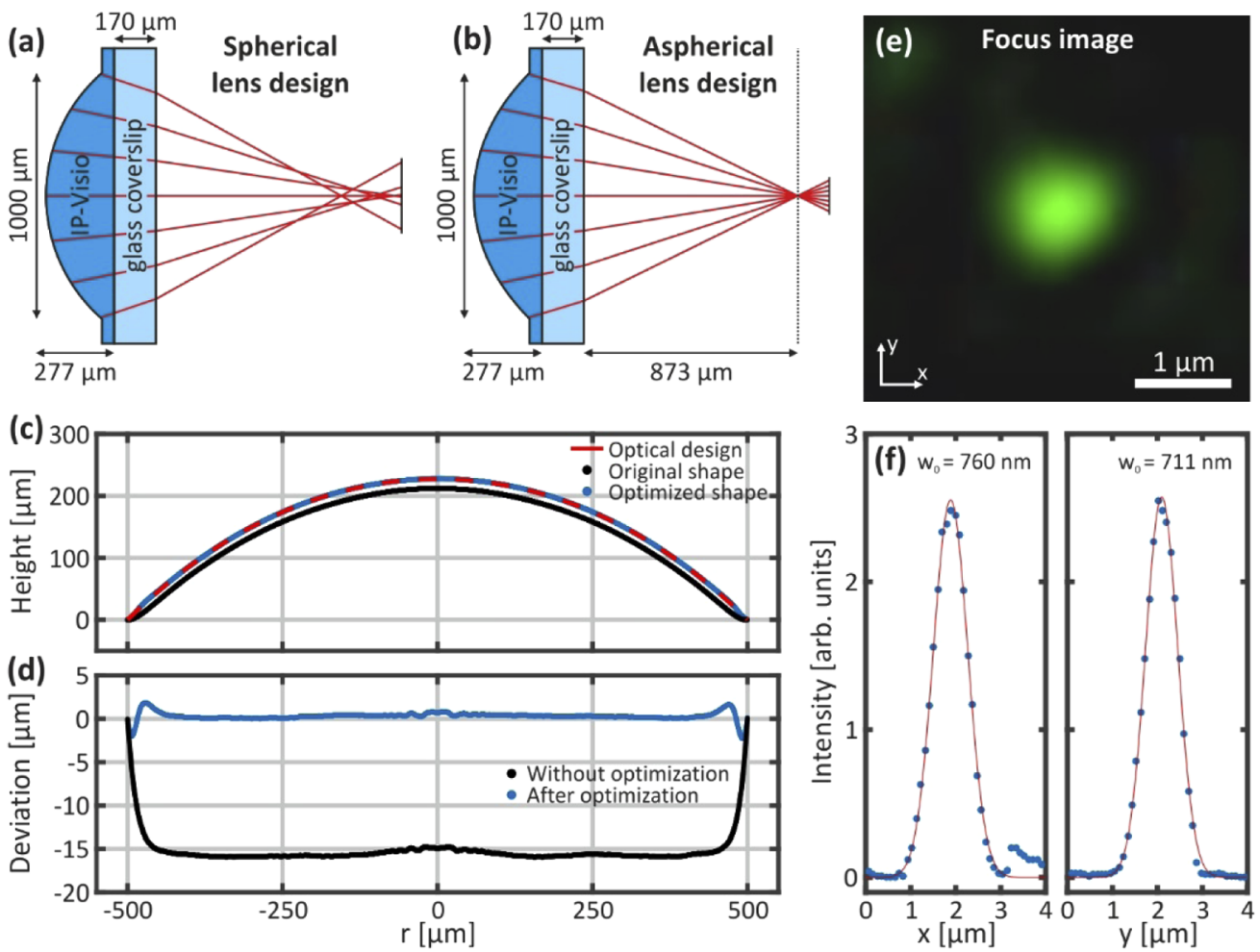

Fig. 2. Design and characterization of aspherical focusing lenses. (a) Optical design of a spherical lens. (b) Aspherical lens design with same diameter and thickness as (a). (c) Lens profile before and after optimization compared to optical design. (d) Shape deviation before and after optimization. (e) Image of focal plane, monochromatic illumination at $550 \mathrm{~nm}$ in combination with aspherical lens from (b). (f) Profiles through (e) in $\mathrm{x}$ and y direction with Gaussian fitting functions.

For further characterization, the focusing capability of the lens is compared to simulations. The lens is designed for an operation wavelength of $550 \mathrm{~nm}$ using the raytracing software ZEMAX OpticStudio. In the optical design the radius of the Airy disk at the focal point is $0.762 \mu \mathrm{m}$. In the experiment, a collimated laser at $550 \mathrm{~nm}$ with a beam diameter of $1 \mathrm{~cm}$ is used for illumination. The beam size is chosen much larger than the lens aperture to ensure homogeneous illumination. The laser is focused by the printed lens $880 \mu \mathrm{m}$ behind the glass coverslip, which is very close to the designed distance of $873 \mu \mathrm{m}$. In the focal plane a weak ring-shaped intensity distribution is visible around the actual spot (Fig. 2(e)). The Gaussian functions fitted to profiles through the focus in $\mathrm{x}$ and $\mathrm{y}$ direction (Fig. 2(f)) have beam radii of $0.760 \mu \mathrm{m}$ in $\mathrm{x}$ direction and $0.711 \mu \mathrm{m}$ in y direction, which is in good agreement with our simulations.

Possible reasons for the ring-shaped halo around the focus spot are the surface roughness of the printed lens, the residual shape deviation, especially in the outer part of the lens, and diffraction at the lens aperture. The bigger deviations at the rim of the lens are most likely caused by shrinking of the flat baseplate. Our optimization algorithm only takes into account the measured height deviation. We assume, however, that there is also a lateral component of the shrinking behavior. A lateral shrinking of the flat ring surrounding the lens could lead to the observed deviations at the transition to the curved lens surface. For a more exact prediction of the shrinking effects the interplay of lateral and perpendicular shrinking should be considered, 
however, this would require the evaluation of numerous test structures of different size and shape and extensive simulations, which would be beyond the scope of this work.

\section{Comparison with glass lenses}

While our aspherical lenses emphasize the design freedom of our 3D printing technique, it is impracticable to compare them directly to similar lenses made from glass, which is the ultimate benchmark material for polymer optics in terms of stability and surface roughness. Aspherical glass lenses with $1 \mathrm{~mm}$ diameter cannot be obtained easily, therefore we print spherical half-ball lenses with diameters of $1 \mathrm{~mm}$ and $2 \mathrm{~mm}$ and compare them to readily available equivalent glass lenses (Edmund Optics).

\subsection{Shape analysis}

Before we compare the optical properties of the lenses we have to verify that both lenses have identical or at least very similar shape. Therefore, we take scanning electron microscopy (SEM) images (Fig. 3(a), (b)). While the glass lens has a perfectly flat bottom and therefore lies tightly on the substrate, a small part at the edge of the printed lens has delaminated from the coverslip. We attribute this to internal tension in the lens caused by shrinking. Furthermore, on the top of the printed lens different slices can be distinguished. This can be expected, as the lens is composed of slices with a finite thickness, resulting in a staircase-shaped surface. For the main part of the lens the difference in diameter between adjacent slices is comparably small and the transition between them is smoothened by the finite voxel size and the proximity effect during the printing process [21]. As the lens surface becomes flatter towards the top, the subsequent slices differ more in diameter, which leads to higher visibility of the distinct steps. While decreasing the slicing distance could reduce this effect, this was omitted here, as it would significantly prolong the writing time.

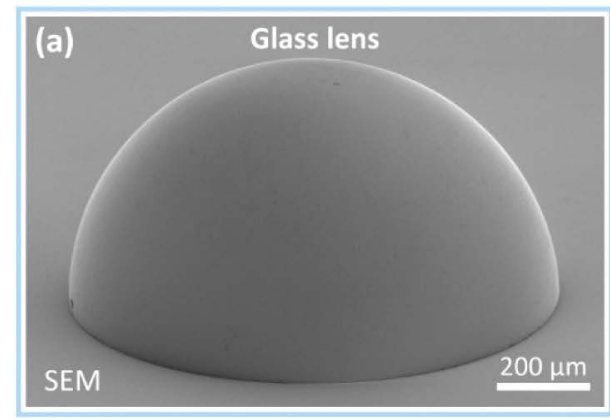

(c)

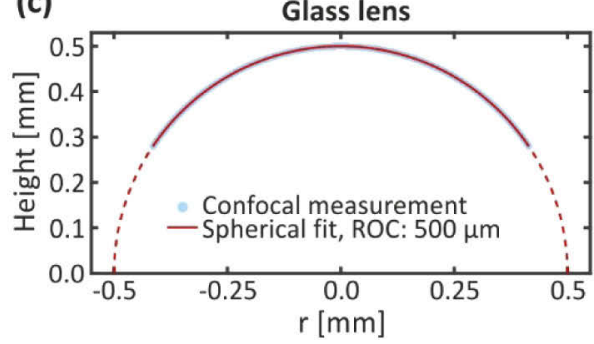

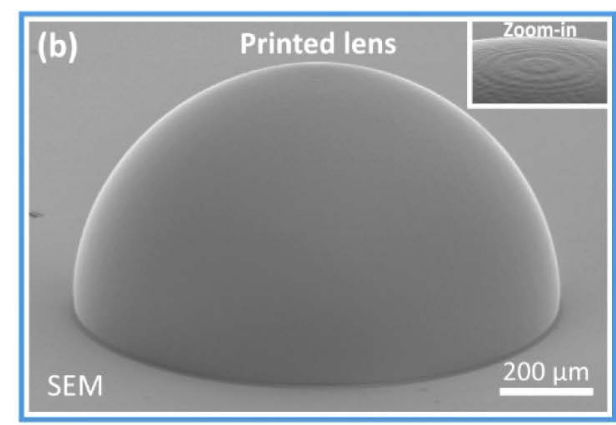

(d)

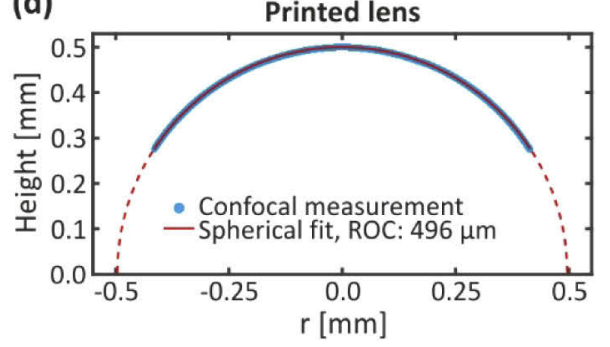

Fig. 3. Half-ball lenses with $1 \mathrm{~mm}$ diameter. (a) SEM image of glass lens. (b) SEM image of 3D printed lens. (c) Profile of glass lens. (d) Profile of 3D printed lens.

The surface profile of both lenses is characterized using a confocal microscopy technique. As the measurement principle relies on light which is directly reflected from the curved lens surface, 
the steeper parts of the lens cannot be measured. The limiting factor is the numerical aperture of the microscope objective (50x, NA 0.9), which is used both for illumination and detection. As a result, the profile measurement is not performed for the total diameter of $1 \mathrm{~mm}$, but only for the upper part of the lens up to a diameter of $0.83 \mathrm{~mm}$ (Fig. 3(c), (d)). Another limitation is the finite working distance of $0.3 \mathrm{~mm}$ of the microscope objective, since it makes it impossible to include the coverslip in the measurement, which is normally used as flat reference plane to compensate for any sample tilt caused by the microscopy setup. Because of these limitations we do not apply the iterative optimization used for the aspherical lenses. Instead, we determine the actual radius of curvature (ROC) of the measured area for both lenses by using a spherical fitting function, plotted in red in Fig. 3(c) and (d). The dashed red line extends the fit to the steeper regions which could not be measured. For the glass lens the ROC is $500 \mu \mathrm{m}$, which perfectly matches the specifications. Due to shrinking, the printed lens has a ROC of $496 \mu \mathrm{m}$, which corresponds to a $0.8 \%$ deviation from the designed $500 \mu \mathrm{m}$.

\subsection{Imaging quality}

Since the shape analysis of the lenses shows only minor differences, it is reasonable to compare them also in terms of imaging quality. The used microscopy setup is sketched in Fig. 4(a) and is similar to the setup published previously [22]. Light from a white LED is collimated by an achromatic lens and illuminates a diffuser plate to ensure homogeneous radiant intensity. It is focused by a microscope objective onto the half-ball lens. A USAF 1951 resolution test target is placed between the objective and the lens. The image of the test target created by the lens is viewed with a standard microscopy setup consisting of objective, tube-lens, and CMOS sensor. As our lenses lack a physical aperture stop to block unwanted stray light, we add an adjustable iris diaphragm between the diffuser plate and the objective. Its position is chosen such that a sharp image of the iris is projected onto the coverslip holding the half-ball lens. The diameter of the image of the diaphragm is then adjusted to match the diameter of the lens, simulating a physical aperture.

The imaging quality of the two lenses is compared in Fig. 4(b) and (c). The object distance between target and half-ball lens is chosen such that the size of the image equals the original size of the test target (1:1 imaging). Exposure time, gain, and white balance settings are identical for both images. The general color impression and contrast is very similar and no visible difference can be seen for groups 4 and 5. When zooming in on the groups 6 and 7 (Fig. 4(d) and (e)), the glass lens shows a slightly better imaging quality, e.g., when looking at elements 4 to 6 of group 7. Reasons for this could be residual deviations from the spherical shape and the surface roughness. Overall, these measurements underline the excellent optical quality and performance of our 3D printed lenses as well as of the lens material.

\subsection{Modulation transfer function}

So far we have studied optics at the $1 \mathrm{~mm}$ scale, yet, our setup allows to push sizes even larger. According to specifications, the utilized 10x microscope objective should only be used for writing field diameters of up to $1 \mathrm{~mm}$ to ensure optimum structural fidelity. This is, however, not the ultimate size limit of our applied configuration. To demonstrate this, we also fabricate a half-ball lens with a diameter of $2 \mathrm{~mm}$ shown in Fig. 5(a). A glass lens is placed next to the printed lens to illustrate the visual similarity of the lens materials. We determine the ROC of the upper part of the printed lens to be $961 \mu \mathrm{m}$, corresponding to a deviation of $3.9 \%$. The spatial root mean square roughness $\mathrm{Sq}$ is $2.9 \mathrm{~nm}$ for the printed lens and $1.9 \mathrm{~nm}$ for the glass lens. In order to get an impression of the full cross-section of the lenses, we tilt the sample by $90^{\circ}$ (Fig. 5(b) and (c)). The horizontal red line indicates the surface of the coverslip and the dashed red line forms a spherical arc with a radius of $1 \mathrm{~mm}$. While the arc overlaps well with the lens boundary of the glass lens in Fig. 5(b), the printed lens in Fig. 5(c) shows deviations from the design. 

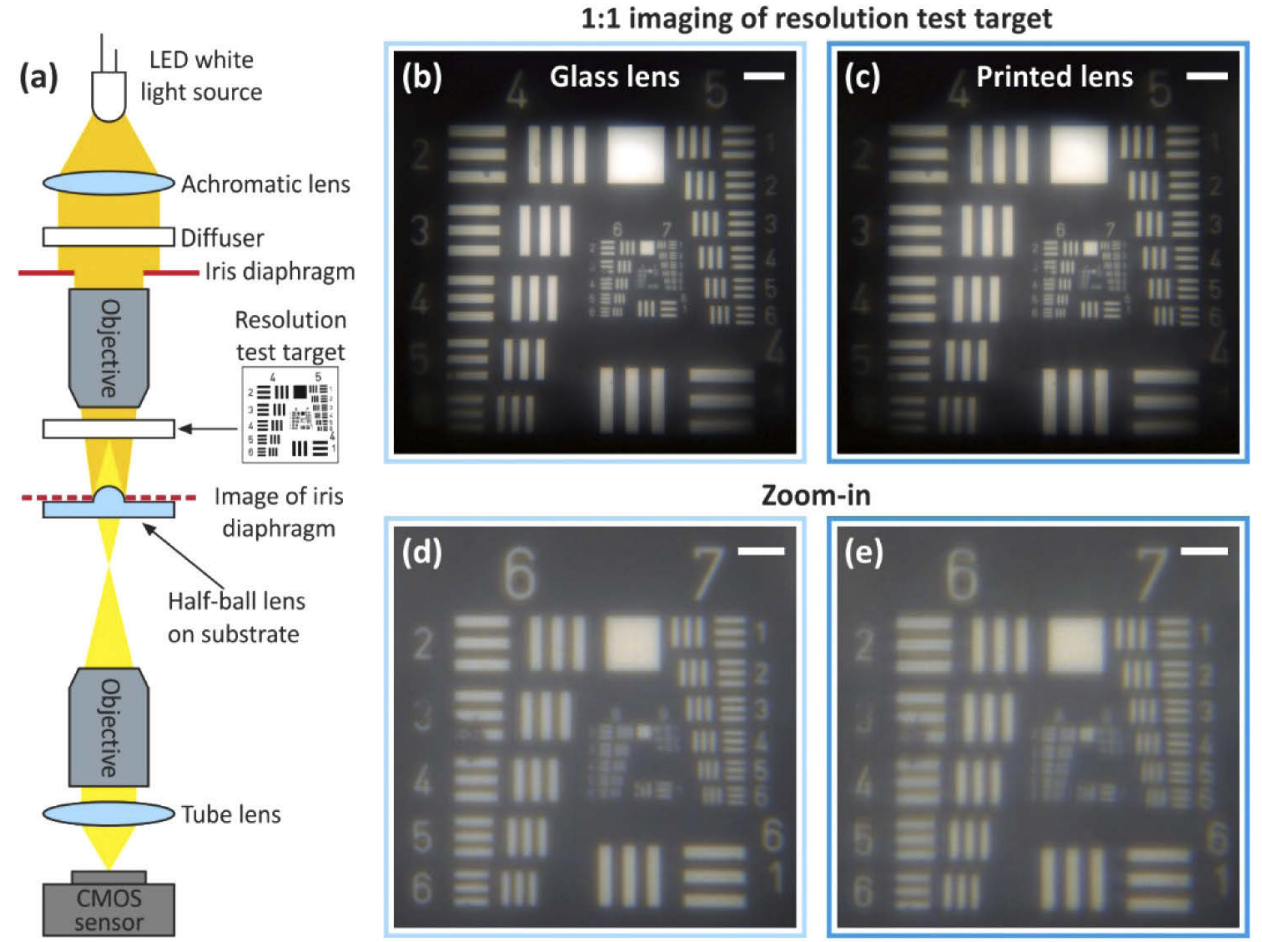

Fig. 4. Imaging quality of half-ball lenses with $1 \mathrm{~mm}$ diameter. (a) Microscopy setup, illumination beam path in dark yellow, detection beam path in light yellow. (b) Resolution test target imaged through glass lens, scale bar: $100 \mu \mathrm{m}$. (c) Resolution test target imaged through printed lens, scale bar: $100 \mu \mathrm{m}$. (d) Zoom-in of (b), scale bar: $30 \mu \mathrm{m}$. (e) Zoom-in of (c), scale bar: $30 \mu \mathrm{m}$.

The lens has delaminated from the substrate at the edges, despite the previous plasma treatment which should ensure good adhesion. Delamination is most probably caused by increased internal tension, resulting from the shrinking of the bigger lens volume. The center-thickness is slightly larger and the lens has a stronger curvature than designed, which is corroborated by confocal microscopy results. Consequently, the shape of glass lens and printed lens is not identical, which has to be considered when comparing the imaging quality of the lenses. We use a commercially available measurement setup (Trioptics Image Master HR) to determine the modulation transfer function (Fig. 5(d)). The MTF of the printed lens falls below 10\% contrast at 210 linepairs $/ \mathrm{mm}$, while this happens only at 290 linepairs $/ \mathrm{mm}$ for the glass lens. We expect the glass lens to have a better MTF in general, which is true for spatial frequencies above 50 linepairs $/ \mathrm{mm}$. The better performance for lower frequencies could result from the delaminated parts of the lens reflecting light away and not contributing to the imaging, as light rays passing the outer sections of a spherical lens are known to deteriorate imaging quality.

Overall, we can conclude that the performance of the 3D printed lens is comparable to the capabilities of the glass lens. As also significant efforts have been invested to optimize commercial glass lenses, we are confident that the shrinkage and additional minimal shape deviations in our 3D printed structures can be overcome as well. Additionally, we again observe that certain tailored deviations can even improve performance. The freedom afforded by 3D printing allows to straightforwardly implement such tailored deviations, underpinning the strength of our ansatz. 

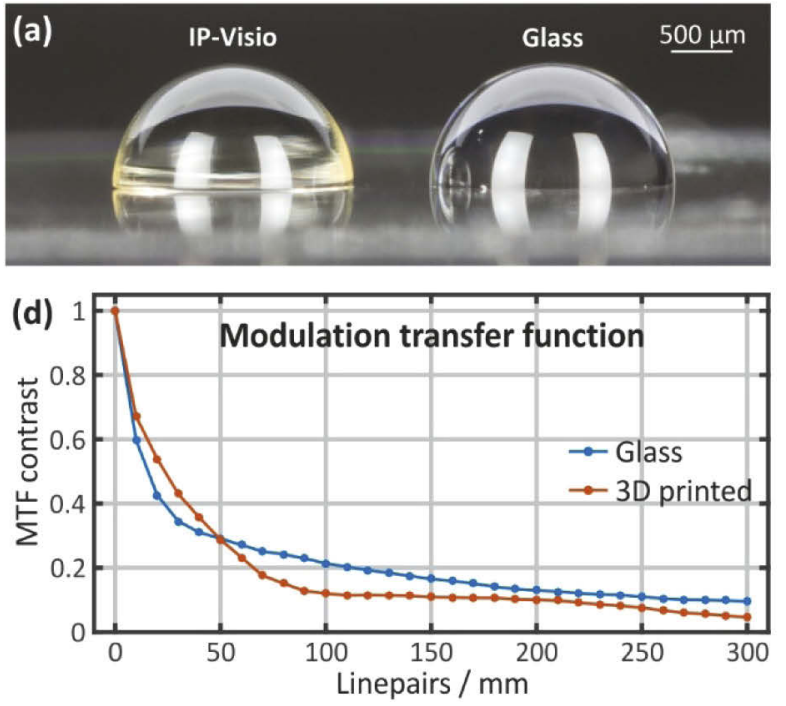
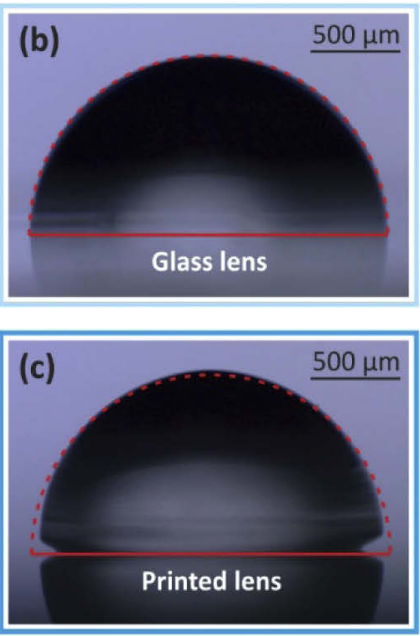

Fig. 5. Half-ball lenses with $2 \mathrm{~mm}$ diameter. (a) Glass lens and printed lens side-by-side. Photographic image by Moritz Flöss. (b) Sied-view of glass lens. (c) Side-view of printed lens. (d) Comparison of modulation transfer function for normal incidence $\left(0^{\circ}\right)$.

\section{Conclusion}

In conclusion, we have successfully fabricated aspherical and spherical lenses with diameters up to $2 \mathrm{~mm}$ by femtosecond 3D printing. Our aspherical lenses are optimized in terms of shape fidelity and have high focusing capability, outperforming commercial spherical glass lenses and illustrating the advantage of free-form 3D printing. Spherical half-ball lenses with $1 \mathrm{~mm}$ diameter show good imaging quality nearly equal to comparable glass lenses. The modulation transfer function of a printed half-ball lens with $2 \mathrm{~mm}$ diameter generally shows a behavior similar to the MTF of the glass counterpart. As they are not optimized, both printed half-ball lenses show certain deviations from the optical design, which are more prominent for the bigger lens. These shape deviations result mainly from the shrinking of the photoresist. The shrinking effects can be effectively counteracted if the lens shape can be characterized accurately. Since this can be challenging for bigger lenses and steeper optical surfaces, new measurement methods will be considered in the future, e.g., X-ray tomography. In order to reduce the residual artifacts caused by slicing and hatching on the lens surface, we will also investigate advanced printing strategies and post-process treatment methods.

Although the lenses studied in this work are basic optical components with a single curved surface, the knowledge of the underlying fabrication process is a crucial first step towards more complex optical systems, e.g., free-hanging lenses with two surfaces or multi-lens objectives for smartphone cameras. Furthermore, we expect the new resist to have a high damage threshold due to its high transparency, which is crucial for high-power applications, e.g., customized optical instruments for medical surgery.

\section{Funding}

Bundesministerium für Bildung und Forschung (PRINTFUNCTION, PRINTOPTICS); European Research Council (AdG COMPLEXPLAS, PoC 3DPRINTEDOPTICS); Deutsche Forschungsgemeinschaft (SPP1839, SPP1929); Baden-Württemberg Stiftung (OPTERIAL); IQST. 


\section{Acknowledgements}

The authors thank Erich Steinbeisser for help with MTF measurements, Moritz Flöss for help with photography and Nanoscribe $\mathrm{GmbH}$ for providing photographic images of the writing objective and the resist bottle. This publication was supported by the Open Access Publishing Fund of the University of Stuttgart.

\section{Disclosures}

The authors declare that there are no conflicts of interest related to this article.

\section{References}

1. T. Gissibl, S. Thiele, A. Herkommer, and H. Giessen, "Sub-micrometre accurate free-form optics by three-dimensional printing on single-mode fibres," Nat. Commun. 7(1), 11763 (2016).

2. A. Heinrich and M. Rank, 3D Printing of Optics (SPIE, 2018).

3. X. Chen, W. Liu, B. Dong, J. Lee, H. O. T. Ware, H. F. Zhang, and C. Sun, "High-Speed 3D Printing of Millimeter-Size Customized Aspheric Imaging Lenses with Sub 7 nm Surface Roughness,” Adv. Mater. 30(18), 1705683 (2018).

4. J. Y. Kim, N. B. Brauer, V. Fakhfouri, D. L. Boiko, E. Charbon, G. Grutzner, and J. Brugger, "Hybrid polymer microlens arrays with high numerical apertures fabricated using simple ink-jet printing technique," Opt. Mater. Express 1(2), 259 (2011).

5. B. G. Assefa, M. Pekkarinen, H. Partanen, J. Biskop, J. Turunen, and J. Saarinen, "Imaging-quality 3D-printed centimeter-scale lens," Opt. Express 27(9), 12630-12637 (2019).

6. T. Gissibl, S. Thiele, A. Herkommer, and H. Giessen, "Two-photon direct laser writing of ultracompact multi-lens objectives," Nat. Photonics 10(8), 554-560 (2016).

7. P. I. Dietrich, M. Blaicher, I. Reuter, M. Billah, T. Hoose, A. Hofmann, C. Caer, R. Dangel, B. Offrein, U. Troppenz, M. Moehrle, W. Freude, and C. Koos, "In situ 3D nanoprinting of free-form coupling elements for hybrid photonic integration," Nat. Photonics 12(4), 241-247 (2018).

8. A. Toulouse, S. Thiele, H. Giessen, and A. M. Herkommer, "Alignment-free integration of apertures and nontransparent hulls into 3D-printed micro-optics," Opt. Lett. 43(21), 5283 (2018).

9. G. von Freymann, A. Ledermann, M. Thiel, I. Staude, S. Essig, K. Busch, and M. Wegener, "Three-dimensional nanostructures for photonics," Adv. Funct. Mater. 20(7), 1038-1052 (2010).

10. J. K. Hohmann, M. Renner, E. H. Waller, and G. von Freymann, “Three-Dimensional $\mu$-Printing: An Enabling Technology," Adv. Opt. Mater. 3(11), 1488-1507 (2015).

11. T. Gissibl, M. Schmid, and H. Giessen, "Spatial beam intensity shaping using phase masks on single-mode optical fibers fabricated by femtosecond direct laser writing," Optica 3(4), 448-451 (2016).

12. J. Li, P. Fejes, D. Lorenser, B. C. Quirk, P. B. Noble, R. W. Kirk, A. Orth, F. M. Wood, B. C. Gibson, D. D. Sampson, and R. A. McLaughlin, "Two-photon polymerisation 3D printed freeform micro-optics for optical coherence tomography fibre probes," Sci. Rep. 8(1), 1-9 (2018).

13. S. Thiele, K. Arzenbacher, T. Gissibl, H. Giessen, and A. M. Herkommer, "3D-printed eagle eye: Compound microlens system for foveated imaging," Sci. Adv. 3(2), e1602655 (2017).

14. Z. C. Ma, X. Y. Hu, Y. L. Zhang, X. Q. Liu, Z. S. Hou, L. G. Niu, L. Zhu, B. Han, Q. D. Chen, and H. B. Sun, “Smart Compound Eyes Enable Tunable Imaging," Adv. Funct. Mater. 29(38), 1903340 (2019).

15. K. Weber, Z. Wang, S. Thiele, A. Herkommer, and H. Giessen, "Distortion-free multi-element Hypergon wide-angle micro-objective obtained by femtosecond 3D printing," Opt. Lett. 45(10), 2784 (2020).

16. S. Fischbach, A. Schlehahn, A. Thoma, N. Srocka, T. Gissibl, S. Ristok, S. Thiele, A. Kaganskiy, A. Strittmatter, T. Heindel, S. Rodt, A. Herkommer, H. Giessen, and S. Reitzenstein, "Single Quantum Dot with Microlens and 3D-Printed Micro-objective as Integrated Bright Single-Photon Source,” ACS Photonics 4(6), 1327-1332 (2017).

17. J. Xu, W. Yao, Z. Tian, L. Wang, K. Guan, Y. Xu, Q.-D. Chen, J.-A. Duan, and H.-B. Sun, "High Curvature Concave - Convex Microlens," IEEE Photonics Technol. Lett. 27(23), 2465-2468 (2015).

18. A. Žukauskas, M. Malinauskas, and E. Brasselet, "Monolithic generators of pseudo-nondiffracting optical vortex beams at the microscale," Appl. Phys. Lett. 103(18), 181122 (2013).

19. L. Jonušauskas, D. Gailevičius, S. Rekštytè, T. Baldacchini, S. Juodkazis, and M. Malinauskas, "Mesoscale laser 3D printing," Opt. Express 27(11), 15205 (2019).

20. M. Schmid, D. Ludescher, and H. Giessen, "Optical properties of photoresists for femtosecond 3D printing: refractive index, extinction, luminescence-dose dependence, aging, heat treatment and comparison between 1-photon and 2-photon exposure," Opt. Mater. Express 9(12), 4564 (2019).

21. E. H. Waller and G. von Freymann, "Spatio-temporal proximity characteristics in 3D $\mu$-printing via multi-photon absorption,” Polymers 8(8), 297 (2016).

22. A. Toulouse, S. Thiele, H. Giessen, and A. M. Herkommer, "Super-fine inkjet process for alignment-free integration of non-transparent structures into 3D-printed micro-optics," Proc. SPIE 10930, 109300W (2019). 